\title{
Sleep Onset Latency
}

National Cancer Institute

\section{Source}

National Cancer Institute. Sleep Onset Latency. NCI Thesaurus. Code C154867.

The duration of time between when the lights are turned off and when the individual falls asleep. 